\title{
Guía clínica para el manejo de la hemorragia subaracnoídea aneurismática - propuesta de actualización al Ministerio de Salud de Chile
}

\author{
Juan Felipe Huidobro Salazar ${ }^{1}$, Leonidas Quintana Marín ${ }^{1,2}$ \\ 1 Neurocirujano Servicio de Neurocirugía ,Hospital Carlos Van Buren, Valparaíso, Chile. \\ 2 Jefe Cátedra de Neurocirugía, Escuela de Medicina ,Universidad de Valparaíso, Chile.
}

\section{Justificación de la guía}

La hemorragia subaracnoídea aneurismática (HSAa) es un problema de salud pública. La alta morbimortalidad y mayor incidencia entre la $5^{\mathrm{a}}$ y $6^{\mathrm{a}}$ década de la vida, generan un alto costo asociado a esta patología, tanto económico, como social, debido a la discapacidad que genera en un segmento productivo de la población ${ }^{1}$.

Durante las últimas décadas, la tendencia de la mortalidad asociada a la HSAa ha sido decreciente ${ }^{2}$. Si bien los factores que han influido en este logro son diversos, se considera que el más importante ha sido la disminución de su letalidad, atribuible a los avances logrados en el manejo médico-quirúrgico durante la etapa aguda de la enfermedad, tanto en el ámbito del tratamiento neurointensivo, como en el de la neurorradiología intervencional y la neurocirugía.

Considerando, por lo tanto, el alto nivel de complejidad que se requiere para manejar esta patología según los estándares actuales, una recomendación transversal en las diversas guías internacionales que tratan sobre HSAa, es la de concentrar los casos en centros especializados, que cuenten con un equipo multidisciplinario disponible para su atención en forma permanente ${ }^{3,4,5}$. Más aún, en dichas guías se establecen algunas condiciones imprescindibles para catalogar un centro como "de referencia". Entre éstas, se encuentra la utilización de guías clíni- cas que estén basadas en la mejor evidencia disponible.

\section{Diagnóstico}

La HSAa es una patología "clásica" de la medicina. Tiene una forma de presentación clínica muy característica, que no debería ser ajena a ningún médico, independiente de su especialidad. Unas de las principales causas de malos resultados en el tratamiento de esta enfermedad, sin embargo, siguen siendo la omisión y la latencia en el diagnóstico de muchos de los casos que se presentan a los servicios de urgencia. Los motivos tras este error diagnóstico en la HSAa han sido objeto de análisis en múltiples publicaciones ${ }^{6}$, que identifican en forma consistente 2 aspectos en los que se generan errores de forma repetitiva: la evaluación clínica y la interpretación de los exámenes diagnósticos.

Si bien es un hecho muy reconocido por los profesionales que se desempeñan en las unidades de urgencia, que el síntoma cardinal de la HSAa es la cefalea ictal, la subvaloración de este síntoma, sobre todo en los casos que se presentan con menor severidad clínica, es algo que ocurre con cierta frecuencia. Una forma de disminuir este error, consiste en mantener un alto índice de sospecha entre el personal que realiza la primera atención, mediante la divulgación y entrenamiento en la utilización de un protocolo de enfrentamiento de la sospecha de HSAa, a partir de la cefalea ictal ${ }^{7}$. Por contraparte, en los casos que se presentan con mayor severidad clínica, los algoritmos de enfrentamiento en la urgencia del compromiso de conciencia, el déficit neurológico focal o la crisis convulsiva, incluyen, generalmente, el estudio precoz del paciente con una tomografía computada (TC) de cerebro, con lo que, la mayoría de las veces, se evita el error diagnóstico. El otro aspecto más asociado a error diagnóstico en la HSAa, la incorrecta interpretación de los exámenes, se refiere específicamente al desconocimiento de las limitaciones de la TC y la punción lumbar (PL). En gran medida, este error podría ser controlado al involucrar tempranamente a los especialistas (neurólogo/neurocirujano) en el proceso diagnóstico, es decir, desde que éste se inicia en la Unidad de Emergencia.

Pese a que más del $80 \%$ de los casos de HSAa se espera que sean pesquisados en forma expedita por los protocolos a los que se hace referencia ${ }^{8}$, se debe tener presente que siempre habrá casos en que el diagnóstico se plantee en forma tardía. Esto se produce más frecuentemente en los casos de presentación atípica, como podría ser un déficit aislado de un $3^{\text {er }}$ par craneano, o cuando se identifica sangre en el espacio subaracnoideo a través de una RM solicitada como estudio diagnóstico en que la sospecha inicial no era la hemorragia subaracnoídea (HSA). El estudio diagnóstico en estos casos, no puede 
ser sistematizado. La mejor aproximación sería, por lo tanto, el manejo individualizado del caso, incluyendo una evaluación conjunta hecha por neurocirujano, neurorradiólogo y neurólogo. Todos los pacientes en que se sospeche una HSA deben ser evaluados por el neurocirujano, quien es el responsable de confirmar o descartar el diagnóstico y, en los casos confirmados, será el encargado de conducir el estudio etiológico.

\subsection{Diagnóstico etiológico}

Una vez confirmado el diagnóstico de HSA, el neurocirujano de turno deberá confirmar o descartar la etiología aneurismática. La importancia de hacer precozmente esta discriminación, radica tanto en la alta morbimortalidad asociada a la etiología aneurismática, como en el hecho de que ésta puede disminuirse significativamente mediante diversos tratamientos, cuya efectividad depende de su implementación oportuna. Entre éstos, por ejemplo, destaca el resangrado, una complicación que generalmente se presenta en las primeras horas de evolución y cuyo mejor tratamiento es la exclusión del aneurisma, ya sea por vía endovascular o quirúrgica.

Al momento de seleccionar el examen para avanzar en el diagnóstico etiológico, se debe considerar que no existe una técnica que sea siempre superior a las demás. La decisión, por lo tanto, depende del análisis de múltiples factores que inciden en el rendimiento del examen y en la probabilidad de complicaciones asociadas. En la Tabla 1, se adjunta una tabla comparativa entre las
3 alternativas diagnósticas, de acuerdo a la realidad actual del Hospital Carlos Van Buren (HCVB) (especificaciones técnicas de los equipos, disponibilidad de especialistas, etc.).

\subsection{Diagnóstico de HSA por reso- nancia magnética (RM)}

Los casos de HSA diagnosticados por RM son esporádicos y, generalmente, de presentación clínica atípica o tardía. Lo anterior, sumado a una significativa probabilidad de encontrar alteraciones en las imágenes secundarias a los procesos fisiopatológicos desencadenados por la misma HSA, como la isquemia cerebral tardía ( $\mathrm{DCl}$, por sus siglas en inglés), por ejemplo, complejiza la interpretación de las imágenes y el diagnóstico en general. Por esta razón, los casos de HSA diagnosticados por esta vía, deben ser evaluados siempre en conjunto con neurorradiología y neurología, tanto para confirmar el diagnóstico, como para definir la necesidad y tipo de estudio etiológico subsecuente.

Cada vez que se decida el estudio por $\mathrm{RM}$, se debe incluir en el examen el estudio vascular (ARM), pues en caso de confirmarse la HSA, esto permitiría utilizar el mismo examen para avanzar en el diagnóstico etiológico.

\subsection{HSA perimescencefálica (HSA- PM)}

Desde sus primeras descripciones a mediados de la década de los 80', los casos de HSA en que la distribución de la sangre en la TC está limitada a las cisternas perimescencefálicas, se han asociado a una historia natural más bien benigna, a una presentación clínica menos severa y a una evolución con menor incidencia de complicaciones que la HSAa. La razón de esto, radica en que la etiología aneurismática es en estos casos muy infrecuente $(2-9 \%)^{9}$.

Por supuesto, la variabilidad de esta cifra depende en gran medida de los criterios que se utilicen para definir la HSA-PM, criterios cobre los cuales no existe actualmente consenso.

Las grandes diferencias que existen entre la HSAa y la HSA-PM en cuanto a historia natural y pronóstico, hacen necesario ajustar las medidas de estudio, monitorización y tratamiento de la HSA-PM, con el fin de hacer más eficiente su manejo, disminuyendo la probabilidad de eventos iatrogénicos, así como también los costos socio-económicos asociados. Dado lo anterior, se recomienda adoptar una definición de HSA-PM e implementar un manejo específico para estos casos.

Tomando en cuenta la evidencia más reciente en cuanto a criterios diagnósticos clínicos e imagenológicos ${ }^{10}$ y la necesidad de excluir efectivamente la etiología aneurismática, a la vez manteniendo una buena correlación diagnóstica interobservador, se establecen los siguientes 7 criterios definitorios de HSA-PM:

- $\quad$ TC de cerebro obtenido $<72$ h desde el inicio del cuadro.

- WFNS 1.

- Centro de la hemorragia contactando la cara anterior del tronco cerebral, en las cisternas prepontina, interpeduncular o supraselar posterior.

- Sangre limitada a las cisternas pre-

Tabla 1.

Comparación de alternativas diagnósticas en HSA

\begin{tabular}{|l|l|l|}
\hline Técnica & Ventajas & Desventajas \\
\hline DSA & $\begin{array}{l}\text { - Máxima sensibilidad y especificidad para el diag- } \\
\text { nóstico de aneurismas, incluso }<3 \mathrm{~mm} \\
\text { - Mejor planificación de tratamiento de aneurismas } \\
\text { complejos } \\
\text { - Potencialmente terapéutica }\end{array}$ & $\begin{array}{l}\text { - Requiere montar pabellón de angiografía } \\
\text { - Utiliza alto volumen de medio de contraste } \\
\bullet \text { Requiere estabilidad hemodinámica }\end{array}$ \\
\hline ATC & $\begin{array}{l}\text { - Rápido } \\
\text { - Accesible } \\
\text { - Buena resolución }\end{array}$ & - Menor especificidad que DSA en aneurismas <3 mm \\
\hline ARM & $\begin{array}{l}\text { No utiliza medio de contraste } \\
\text { - Requiere estabilidad clínica vs utilización de anestesia } \\
\text { general }\end{array}$ \\
\hline DSA angiografía cerebral, ATC angiotomografía, ARM angiorresonancia.
\end{tabular}


pontina, interpeduncular, supraselar, crural, ambient, y/o cuadrigeminal, y/o magna.

- Sangre intraventricular limitada al llene parcial del cuarto ventrículo y los cuernos occipitales de los ventrículos laterales.

- Ausencia de sangre en fisuras silviana o interhemisférica.

- Ausencia de sangre intraparenquimatosa.

Para que un caso sea definido como HSA-PM, deberá cumplir con todos los criterios. Además, se deben excluir aquellos casos que, en su evolución, presenten $\mathrm{DCl}$ o resangrado.

Pese a lo anterior y considerando los recursos imagenológicos actualmente disponibles en el HCVB, la HSA-PM debe ser estudiada, siempre que sea posible, mediante angiografía cerebral (DSA). En caso de no identificarse aneurismas en la DSA, las recomendaciones de diagnóstico, monitorización y tratamiento propuestas en la presente guía (ver más adelante), deberían ser ajustadas. En este sentido, no se considerará imprescindible la repetición del estudio angiográfico, pudiendo ser utilizadas en su reemplazo técnicas no invasivas, como el ATC y la ARM. Del mismo modo, la intensidad de la monitorización y la agresividad de los tratamientos, deberían ser menores.

\subsection{Algoritmo de enfrentamiento} diagnóstico de la HSAa (Figura 1)

El proceso diagnóstico se inicia en la Unidad de Emergencia Adulto (UEA), en el momento en que el cuadro clínico obliga al médico a considerar la HSAa dentro de su diagnóstico diferencial. EI objetivo central del algoritmo, es disminuir al máximo la posibilidad de omitir el diagnóstico de HSAa y, al mismo tiempo, hacerlo de la forma más expedita posible. En este sentido, es necesario aclarar que este algoritmo no pretende ser una guía para el enfrentamiento general de la cefalea en la UEA.

El algoritmo asume como punto de inicio la cefalea ictal y establece la TC de cerebro sin medio de contraste (TC $\mathrm{s} / \mathrm{c}$ ) como el primer test diagnóstico a aplicar. Se asume, sin embargo, que la HSA también puede ser pesquisada en una TC s/c solicitada en el contexto de flujogramas paralelos, como sería el caso en un déficit focal de reciente inicio, una primoconvulsión o el compromiso de conciencia. En estos casos,

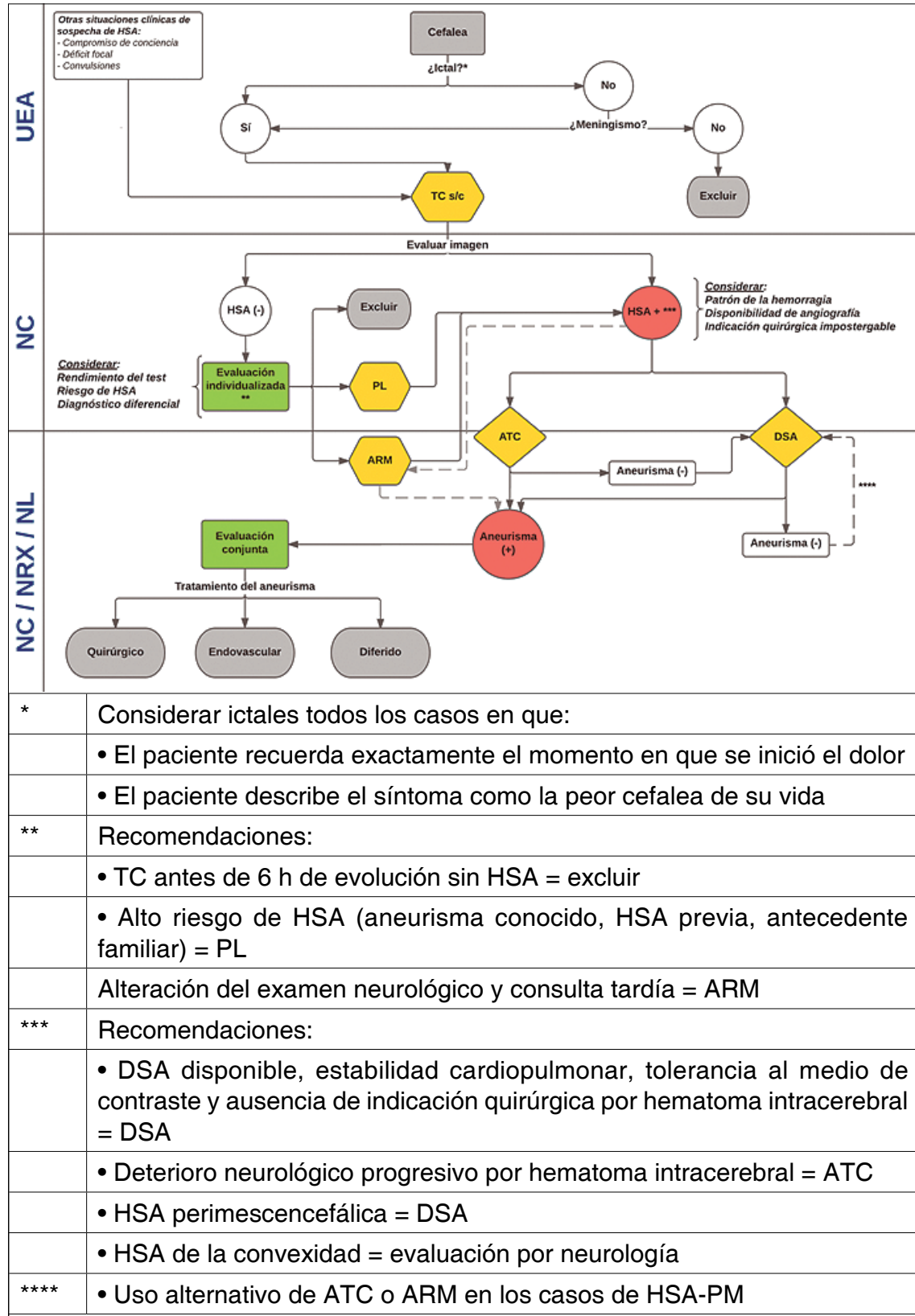

TC s/c scanner de cerebro sin contraste, HSA hemorragia subaracnoídea, PL punción lumbar, ARM resonancia magnética de cerebro con angiorresonancia, ATC angiotomografía, DSA angiografía cerebral.

Figura 1. Algoritmo diagnóstico de la HSA.

tras ser evaluada la imagen por el neurocirujano de turno, continúan el algoritmo diagnóstico de HSAa sólo aqueIlos pacientes en que la TC s/c muestra HSA y se excluyen los demás.

Ante una TC s/c negativa, no existe actualmente consenso en cuanto a cómo proseguir el estudio ${ }^{11}$. Tradicionalmente se ha considerado el estudio de líquido cefalorraquídeo (LCR) mediante punción lumbar $(\mathrm{PL})$ como el "estándar de oro" para el diagnóstico de HSA. Esta conducta, sin embargo, ha sido cuestionada en diversas publicaciones recientes $^{12}$, tanto por la baja probabilidad de HSA en un paciente de bajo riesgo con TC s/c negativo ${ }^{13}$, como por la falta de consenso en cuanto a los criterios de análisis e interpretación del examen (manipulación de la muestra, 
uso de espectrofotometría, etc.) y que se sabe inciden directamente en el rendimiento del mismo ${ }^{14}$.

Considerando que el debate al respecto está abierto, y teniendo en cuenta los elementos de apoyo diagnóstico actualmente disponibles en el HCVB (carencia de espectrofotometría para el análisis del LCR, disponibilidad de resonancia magnética, entre otros), no es posible establecer una conducta única en cuanto a la mejor secuencia de estudio. Se sugieren, en cambio, 3 alternativas posibles. La decisión entre estas alternativas, debe tomarse tras el análisis del caso particular. Para esto, en la reseña de la Figura 1 se señalan los factores indispensables a considerar y se hace una recomendación para algunos contextos clínicos en que, a priori, una conducta sería más apropiada que las otras. En cuanto al estudio etiológico, las recomendaciones están basadas en las características de los exámenes detalladas en la Tabla 1.

\section{Categorización}

De acuerdo a las concepciones más modernas de la enfermedad, el pronóstico de la HSAa, cuando es manejada bajo los estándares actuales (tratamiento precoz del aneurisma o la hidrocefalia, por ejemplo), está determinado fundamentalmente por 2 factores: la injuria cerebral precoz (EBI, por sus siglas en inglés) y la $\mathrm{DCl}$.

El concepto de EBI, englobaría múltiples mecanismos fisiopatológicos iniciados por la isquemia cerebral global, secundaria al aumento brusco de la presión intracraneana (PIC) en el momento de la ruptura aneurismática. La magnitud de este daño, podría ser estimada clínicamente, por ejemplo, por el compromiso de conciencia inicial no atribuible a otras causas, como hidrocefalia o estatus epiléptico. Si bien la $\mathrm{EBI}$ no es un elemento modificable con el tratamiento, su estimación es muy importante del punto de vista clínico, puesto que permitiría aproximarse mejor al pronóstico del paciente y ajustar el nivel de monitorización necesario para cada caso, pues, a mayor carga de EBI, mayor susceptibilidad del paciente a los efectos deletéreos de la $\mathrm{DCl}$.

A diferencia de lo que sucede con la $\mathrm{EBI}$, el desarrollo de $\mathrm{DCl}$ se produce tardíamente y es, por lo tanto, en cierta medida susceptible de tratar. Desde esta perspectiva, la mejor oportunidad de seguir disminuyendo la tasa de morbimortalidad asociada a la HSAa, está en la optimización del manejo de la DCl. Esta situación, ha convertido a la $\mathrm{DCl}$ en uno de los principales focos de interés de la HSAa para la comunidad científica, lo que se ha traducido en grandes avances en los últimos años, sobre todo en cuanto a su definición y fisiopatología.

Teniendo en cuenta lo anteriormente expuesto, se puede entender la importancia de una buena aproximación al pronóstico de cada paciente. Es por esta razón, que desde hace varias décadas se ha intentado estratificar a los pacientes con HSAa según el riesgo de complicaciones y de mal outcome. Para esto, se han elaborado múltiples escalas de clasificación. Entre éstas, se recomienda utilizar las 3 que se presentan a continuación. Estas escalas deben ser aplicadas en todos los casos de HSAa.

\subsection{World Federation of Neurosurgi- cal Societies (WFNS)}

Consiste en una clasificación de los pacientes según la severidad de la presentación clínica. Ha demostrado una asociación directa con la morbimortalidad $^{15}$.

Si bien existen otras escalas similares, algunas ampliamente difundidas y empleadas, como la de Hunt y Hess, se recomienda la WFNS por sobre otras debido a: una buena correlación interobservador, atribuible a la utilización de una terminología más objetiva ${ }^{16}$; una adecuada correlación entre mayor grado y peor pronóstico ${ }^{15}$; amplia utilización a nivel mundial e incorporación en el sistema VASOGRADE.

Pese a que no existe consenso respecto al momento en que debe ser aplicada esta escala, en los trabajos más recientes relacionados a HSAa se ha utilizado sólo después de la reanimación inicial ${ }^{16} y$, en el caso de pacientes con HSAa de alto grado en que se decida instalar una derivación ventricular externa (DVE), después de que ésta ha sido instalada ${ }^{17}$ En la Figura 2, se muestra la clasificación de la WFNS, extraída de la publicación original ${ }^{18}$.

\subsection{Escala de Fisher modificada (EFM)}

Tal como sucede con la clasificación de la severidad clínica, son múltiples las escalas que se han propuesto para estimar la probabilidad de ocurrencia de DCl. Muchas de ellas, se basan en una conocida asociación directa entre distribución/cantidad de sangre y riesgo de vasoespasmo. Por otra parte, se han propuesto además muchas otras, que consideran también parámetros clínicos.

Entre todas, se recomienda la mFS (Figura 3, extraída de la publicación

\begin{tabular}{|l|}
\hline Escala de graduación de la HSA (según la WFNS) \\
\hline Grado 0: Glasgow 15; sin HSA \\
\hline Grado I: Glasgow 15; sin déficit focal \\
\hline Grado II : Glasgow 13-14; sin déficit focal \\
\hline Grado III: Glasgow 13-14; con déficit focal \\
\hline Grado IV: Glasgow 7-12; con o sin déficit focal \\
\hline Grado V: Glasgow 3-6; con o sin déficit focal \\
\hline
\end{tabular}

Figura 2.

\begin{tabular}{|l|}
\hline Escala de Fisher modificada \\
\hline Grados \\
\hline 0 - No HSA o hemorragia intraventricular \\
\hline 1 - HSA focal o difusa fina, sin hemorragia intraventricular \\
\hline 2 - HSA focal o difusa fina, con hemorragia intraventricular \\
\hline 3 - HSA focal o difusa gruesa, sin hemorragia intraventricular \\
\hline 4 - HSA focal o difusa gruesa, con hemorragia intraventricular \\
\hline
\end{tabular}

Figura 3. 
original ${ }^{19}$ ) porque es más simple, tiene mejor concordancia interobservador, ha sido sometida a una validación prospectiva respecto al riesgo de $\mathrm{DCl}^{19}$ y porque está incorporada en el sistema VASOGRADE.

\subsection{VASOGRANDE}

En la misma línea de intentar predecir la $\mathrm{DCl}$, pero con una aplicación clínica mayor, se han diseñado recientemente otros sistemas de clasificación. Entre éstos, se recomienda el VASOGRADE porque: ha demostrado una asociación directa con el riesgo de $\mathrm{DCl}$; es sencillo y reproducible, pues utiliza WFNS y $\mathrm{mFS}$; existe experiencia favorable en su utilización para definir intensidad de monitorización y períodos de hospitalización.

En la Figura 4, se muestra la clasificación según VASOGRADE, extraída de la publicación original ${ }^{17}$.

\section{Monitorización}

El paciente con HSAa está sometido al riesgo de una serie de complicaciones conocidas, que se presentan con mayor probabilidad en períodos específicos de su evolución. Así, la morbimortalidad asociada a la HSAa, depende de la adecuada implementación de medidas que disminuyan la incidencia de dichas complicaciones, que permitan la pesquisa precoz de su presentación y que faciliten en forma oportuna y efectiva los tratamientos de rescate disponibles.

Una forma práctica de disminuir la morbimortalidad de una manera eficiente, por lo tanto, consiste en protocolizar el tipo e intensidad de la monitorización de acuerdo al riesgo de complicaciones del paciente y sistematizar el enfrentamiento a partir, principalmente, de la identificación del deterioro neurológico. Si bien ante la aparición del deterioro neurológico es necesario considerar todas las posibles causas, el tiempo de evolución, el contexto clínico y la forma de presentación deben orientar la secuencia de exámenes que se soliciten para identificarla. Así, por ejemplo, la intensificación progresiva de la cefalea asociada a un deterioro rápido del nivel de conciencia en las primeras horas del cuadro, es con mayor probabilidad la presentación de una hidrocefalia aguda y debería ser evaluada inmediatamente con un TC de cerebro. Por otra parte, el hallazgo de una mínima paresia braquial, en el contexto de una disminución del nivel de atención o de fluencia del lenguaje en el $7^{\circ}$ día, en cambio, es más probablemente la manifestación de $\mathrm{DCl}$, un diagnóstico que no puede confirmarse sino tras haber descartado todas las demás causas, sistémicas y neurológicas, de deterioro neurológico. En la Figura 5, se muestra un resumen de las medidas generales de tratamiento, el nivel de monitorización según VASOGRADE y el enfrentamiento inicial ante la identificación de deterioro neurológico y, en particular, de $\mathrm{DCl}$.

Como se desprende de la Figura 5, el nivel de vigilancia que se requiere para manejar un paciente con HSAa, hace imprescindible su hospitalización inmediata en una unidad de paciente crítico (UPC).

\section{Enfrentamiento de las complica- ciones neurológicas}

\subsection{Compromiso de conciencia}

Si bien la alteración cuali-cuantitativa de conciencia es muchas veces sólo la manifestación clínica de los procesos fisiopatológicos propios de la HSAa (EBI), una alternativa es que, al menos en parte, este compromiso neurológico sea secundario a alguna complicación asociada que pueda ser tratada, con la consecuente mejoría neurológica. El enfrentamiento del compromiso de

\begin{tabular}{|l|c|l|}
\hline VASOGRADE & ESCALA WFNS & FISHER MODIF. \\
\hline VERDE & $1-2$ & $1-2$ \\
\hline \hline AMARILLO & $1-3$ & $3-4$ \\
\hline \hline ROJO & $4-5$ & CUALQUIERA \\
\hline \multicolumn{2}{|l}{ Figura 4. Clasificación VASOGRADE } \\
\hline
\end{tabular}

conciencia, por lo tanto, no difiere de lo propuesto en la Figura 5 respecto al deterioro neurológico. El motivo de su descripción como una complicación neurológica específica, por lo tanto, se refiere a la situación particular en que ésta no sea atribuible a hidrocefalia, alteraciones hidroelectrolíticas, compromiso cardiopulmonar o estatus epiléptico. En otras palabras, se refiere a los verdaderos casos de HSAa de alto grado (VASOGRADE rojo).

El mal pronóstico general del paciente con HSAa de alto grado, incluso muchas veces pese a un manejo integral óptimo de la patología, hace necesaria la consideración de múltiples factores para determinar la pertinencia de la hospitalización en la Unidad de Cuidados Intensivos (UCI) y la consiguiente implementación de medidas invasivas. Entre estos factores, se deben considerar la edad avanzada, la presencia de un aneurisma complejo, las patologías concomitantes, el estado funcional previo y las preferencias de la familia. En el caso de los pacientes con HSAa

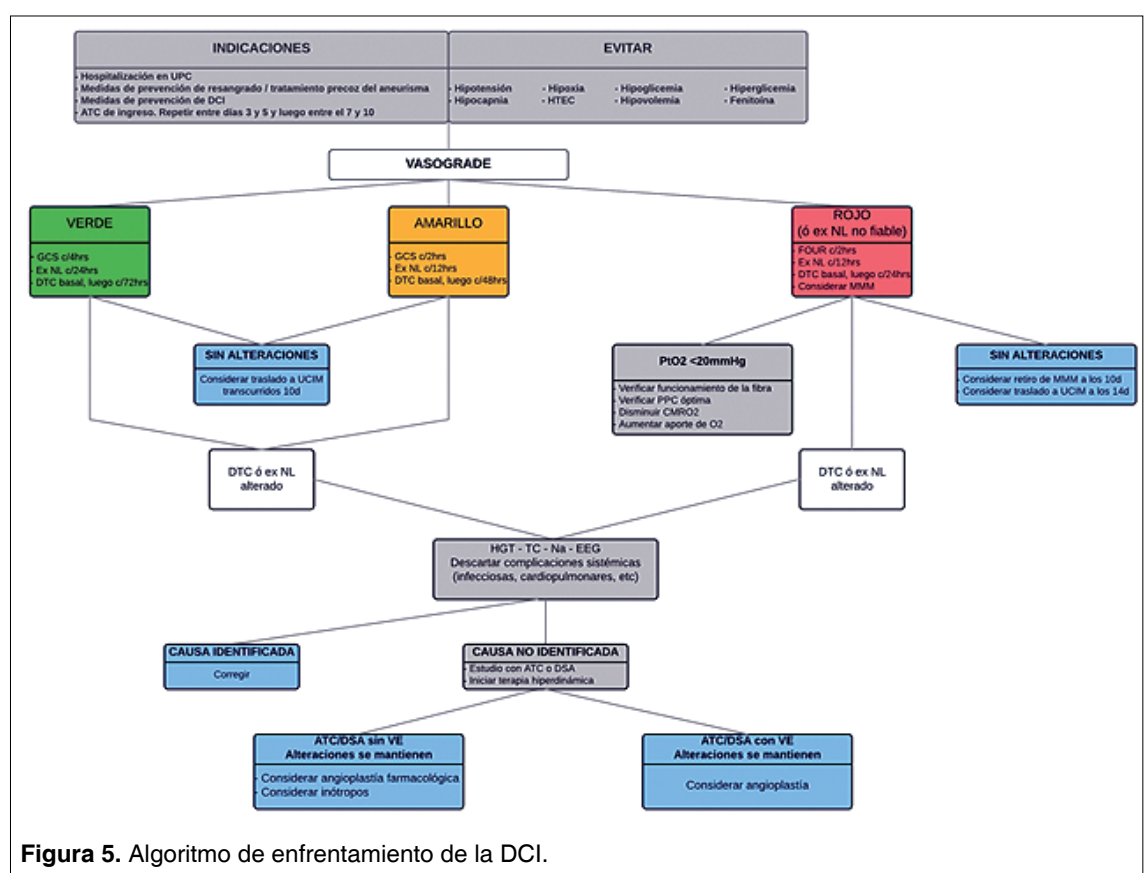


de alto grado que son hospitalizados en $\mathrm{UCl}$, y en todos los demás casos en que la evaluación neurológica esté comprometida (necesidad de sedación profunda para acoplamiento a VMI, agitación, etc.) se debe considerar la implementación de todas las medidas de monitorización multimodal (MMM) disponibles para orientar el manejo del paciente, incluyendo las invasivas (PIC, PtO2, SjO2). Pese a que no existe evidencia robusta de la utilidad de cada una de estas mediciones por separado en el outcome clínico de los pacientes, la tendencia actual está inclinada fuertemente a su utilización, pues permitiría una mejor comprensión de los fenómenos fisiopatológicos involucrados y, de esta forma, un tratamiento individualizado, específico para cada situación. Además, la MMM tendría un rol fundamental en la vigilancia, la evaluación de la respuesta a las medidas terapéuticas y la determinación del pronóstico de los pacientes ${ }^{20,21}$.

\subsection{Resangrado}

La posibilidad de resangrado de un aneurisma cerebral, debe ser una de las principales consideraciones durante el manejo inicial de la HSAa. El riesgo de ocurrencia de esta complicación es mayor en las primeras horas, pero sigue presente en las siguientes semanas, mientras no se haya excluido el aneurisma. Dado que la mortalidad asociada al resangrado es cercana al $70 \%$, se recomienda tratar el aneurisma lo antes posible.

En los casos de HSAa de bajo grado (WFNS 1, 2 y 3), el tratamiento del aneurisma debe realizarse antes de 24 $\mathrm{h}^{1}$ a partir del momento en que se confirma el diagnóstico de HSAa.

En los casos de HSAa de alto grado (WFNS 4 y 5), el mejor timing para el tratamiento del aneurisma debe ser discutido caso a caso por un equipo compuesto por neurocirujano, neurorradiólogo intervencional e intensivista. En los casos en que se decida diferir el tratamiento del aneurisma, se debe considerar el tratamiento con ácido tranexámico $1 \mathrm{gr}$ cada $6 \mathrm{~h}$ hasta la exclusión aneurismática o, máximo, por 72 $\mathrm{h}^{22}$.

El tratamiento del aneurisma, ya sea por vía quirúrgica o endovascular, es un tratamiento de alta complejidad y, por lo tanto, tiene una latencia insoslayable de algunas horas hasta su concreción. Esto significa que, durante el período de mayor riesgo de re-ruptura aneurismática, se deben implementar una serie de medidas agregadas para disminuir dicho riesgo. Estas medidas tienen como objetivo el control de la presión arterial (PA), de la agitación y del dolor.

\subsubsection{Control de la PA}

Se recomienda el control de la PA desde el diagnóstico en UEA con fármaco(s) titulable(s), para disminuir el riesgo de resangrado, aunque sin comprometer la perfusión cerebral, buscando una $\mathrm{PAS}<160$ o $\mathrm{PAM}<110$. Considerando el arsenal farmacológico actualmente disponible en el HCVB, se recomienda la administración de labetalol en bolo $5-20 \mathrm{mg}$ ev, seguido por BIC 0,5-2 $\mathrm{mg} / \mathrm{min}^{23}$.

\subsubsection{Analgesia}

La cefalea severa es un síntoma frecuente y de difícil tratamiento en la HSAa, sin embargo, no se dispone actualmente de guías que se refieran específicamente a su manejo ${ }^{24}$. Los principios que justifican la importancia de su tratamiento, más allá de la evidente necesidad de proporcionar alivio al paciente, son facilitar el control de la PA y la agitación y evitar la hipoperfusión cerebral por HTEC secundaria.

Se recomienda para esto, ajustar la analgesia a la intensidad del síntoma, considerando para el tratamiento de la cefalea severa la asociación de múltiples fármacos y la utilización temprana de algún analgésico de mayor potencia, como la morfina o el fentanil, procurando evitar dosis que alteren la fidelidad del examen neurológico.

\subsubsection{Control de la agitación}

La indicación de mantener al paciente con una HSAa en reposo absoluto en $30^{\circ}$, además de disminuir la posibilidad de una re-ruptura aneurismática por una cuestión mecánica, ya sea por el movimiento en sí o por la elevación secundaria de la PA, se fundamenta en el beneficio teórico de favorecer el acoplamiento neurovascular, por ejemplo, disminuyendo el consumo metabólico cerebral y favoreciendo el flujo sanguíneo cerebral (FSC) mediante la disminución de la PIC.

Al igual que lo que sucede con el manejo del dolor, la evidencia actual no permite hacer una recomendación específica al respecto, por lo que sólo se sugiere fuertemente considerar los conceptos previamente descritos al momento de tratar al paciente con HSAa.

\subsection{Hidrocefalia}

La hidrocefalia es una complicación frecuente de la HSAa, estimándose una incidencia de alrededor del $20 \%$, siendo la mitad de estos casos de presentación precoz y evolución rápidamente progresiva. El otro $50 \%$ correspondería al grupo tradicionalmente considerado como hidrocefalia reabsortiva. Al respecto, se hacen las siguientes recomendaciones:

- En los casos que presentan deterioro neurológico desde el ingreso, o que lo desarrollan precozmente ( $<72 \mathrm{~h}$ de inicio del cuadro), y que presentan dilatación del sistema ventricular en el TC, se debe considerar la instalación inmediata de una DVE, independiente de la presencia de un aneurisma no tratado.

- En los pacientes neurológicamente estables y con aneurisma tratado, en que se instaló una DVE, se debe considerar el weaning precoz de ésta (24 a $72 \mathrm{~h}$ ).

- Los casos que evolucionen con hidrocefalia reabsortiva, deben ser tratados mediante la instalación de una derivación ventrículo-peritoneal (DVP) en cuanto las condiciones lo permitan.

\subsection{Convulsiones}

Pese a que las convulsiones son una complicación reconocida de la HSAa, en los últimos años ha habido un cambio en el paradigma de su enfrentamiento atribuible, principalmente, a la publicación de evidencia que mostró la asociación entre el uso de fenitoína profiláctica y peores resultados cognitivos $^{25}$. Desde entonces, las distintas guías de manejo de HSAa recomiendan evitar el uso de fenitoína profiláctica. Lo anterior, no significa que no esté recomendado el tratamiento con fármacos anti-epilépticos, pues se sabe que el paciente que ya presentó un episodio convulsivo tiene un riesgo aumentado de un nuevo episodio, con el consiguiente riesgo de resangrado y desacoplamiento neurovascular. Además, la carga de episodios epileptiformes durante la evolución de un paciente con HSAa, también tendría una 
asociación directa con un peor resultado cognitivo y funcional ${ }^{26}$. En virtud de lo anterior, la recomendación actual es evitar la profilaxis con fenitoína, pero tratar activamente, ya sea con fenitoína o levetiracetam, a los pacientes en que exista evidencia clínica o electroencefalográfica contundente de actividad epiléptica.

Tomando en consideración toda la evidencia actualmente disponible, se propone la siguiente recomendación local para facilitar la toma de decisión al respecto:

- Paciente CON crisis epilépticas clínicas o eléctricas (EEG):

Inicio: Tratamiento con $2 \mathrm{gr}$ de levetiracetam endovenoso o enteral seguido, a las $8 \mathrm{~h}$, de $1,5 \mathrm{gr}$ cada $12 \mathrm{~h}$. En caso de contraindicación o no disponibilidad de levetiracetam, administrar fenitoína $20 \mathrm{mg} / \mathrm{kg}$ en carga endovenosa y continuar mantención según peso estimado del paciente. Controlar con niveles plasmáticos lo antes posible.

Discontinuación: Una vez controlado el cuadro epiléptico y según el potencial epileptogénico de las lesiones estructurales, entre 1 a 3 meses.

- Paciente SIN crisis epilépticas clínicas ni eléctricas (EEG):

Inicio: Profilaxis con 2 gr de levetiracetam endovenoso o enteral seguido, a las $8 \mathrm{~h}$, de $1 \mathrm{gr}$ cada 12 h. En caso de contraindicación o no disponibilidad de levetiracetam, administrar fenitoína $20 \mathrm{mg} / \mathrm{kg}$ en carga endovenosa y continuar mantención según peso estimado del paciente. Controlar con niveles plasmáticos lo antes posible.

Discontinuación: a las 72 h en los casos de exclusión aneurismática endovascular y a los 7 días en los casos de exclusión aneurismática quirúrgica.

\subsection{Isquemia cerebral tardía (DCI)}

A la luz de los últimos avances en el estudio de los fenómenos fisiopatológicos desencadenados por la HSA, el concepto de $\mathrm{DCl}$, como entidad nosológica, ha sido bastante cuestionado. Se le critica una definición confusa y el hecho de ser un concepto ambiguo, que incluye mecanismos fisiopatológicos muy distintos ${ }^{20}$. Esto se puede ver reflejado en la heterogeneidad de los criterios utilizados para diagnosticar la $\mathrm{DCl}$ en los distintos trabajos de inves- tigación. Por otra parte, esta situación podría explicar, en cierta medida, el mal resultado que han tenido los últimos tratamientos que se han probado para mejorar los outcomes funcionales en pacientes que evolucionan con $\mathrm{DCl}$. Pese a lo anterior, el término $\mathrm{DCl}$ sigue siendo universalmente utilizado para explicar el deterioro neurológico o infarto cerebral de un paciente cursando una HSA, cuando no se encuentra una causa alternativa.

Dado que el vasoespasmo (VE) ha sido tradicionalmente el principal mecanismo fisiopatológico al que se ha atribuido la $\mathrm{DCl}$, el eje central del manejo de la $\mathrm{DCl}$ consiste en la prevención, pesquisa precoz y tratamiento del VE.

En la presente guía, los conceptos de $\mathrm{DCl}$ y $\mathrm{VE}$ se emplean de manera convencional, en acuerdo con los últimos consensos internacionales ${ }^{27}$, y los tratamientos recomendados incluyen sólo aquellos para los que existe actualmente evidencia que los respalde. Se sugiere, sin embargo, en el tratamiento individualizado de los casos, considerar la utilización de medidas terapéuticas alternativas, en la medida en que la racionalidad, determinada por la interpretación de los mecanismos fisiopatológicos subyacentes a la $\mathrm{DCl}$ en un paciente en particular, así lo dicte.

A continuación, se presentan las definiciones y los tratamientos recomendados. El algoritmo diagnóstico y terapéutico que se sugiere a partir de esto, se muestra en la Figura 5.

\section{Definiciones:}

- $\quad D C l$ : aparición de déficit neurológico focal (hemiparesia, afasia, negligencia, apraxia, hemianopsia) y/o caída de 2 puntos o más en la escala de coma de Glasgow (GCS), de > 1 de duración, habiéndose excluido otras causas y que no se presenta inmediatamente después de la exclusión aneurismática. Alternativamente, la $\mathrm{DCl}$ se puede diagnosticar en forma retrospectiva, mediante la pesquisa de lesiones isquémicas nuevas en TC o RM, dentro de 6 semanas de iniciado el cuadro y que no se observaban en las primeras $48 \mathrm{~h}$ del tratamiento del aneurisma.

- VE: disminución del calibre de las arterias intracraneanas del polígono de Willis de $>50 \%$, demostrado por DSA o ATC 28 .
- Sospecha de VE: puede ser diagnosticado por doppler transcraneano (DTC) según los siguientes criterios: aumento de la velocidad media (del flujo en la arteria cerebral media $(A C M)>50 \mathrm{~cm} / \mathrm{s}$ en $<$ $24 \mathrm{~h}$ o velocidad media $>200 \mathrm{~cm} / \mathrm{s}$ o > $120 \mathrm{~cm} / \mathrm{s}$ con índice de Lindegaard > 3 . En pacientes sedados, con MMM, se debe considerar también el diagnóstico con PtO2 $<20$ $\mathrm{mmHg}$ o $\mathrm{SjO} 2<50 \%$, tras haber optimizado el manejo de PIC, PPC y acoplamiento neurovascular. Alternativamente, se debe considerar la utilización de estudios de estimación del flujo sanguíneo cerebral, como la RM perfusión $\mathrm{y}$, de estar disponible, el CT perfusión, que es actualmente la herramienta más práctica para este propósito y que sería recomendable incorporar en nuestro centro.

\subsubsection{Prevención de la $\mathrm{DCl}$ \\ 5.5.1.1 Euvolemia}

Se recomienda que el paciente con HSAa se mantenga con una volemia adecuada para mantener el flujo sanguíneo cerebral, dado que existe evidencia que muestra peores resultados atribuibles a hipo e hipervolemia (Figura 6$)^{29}$. El manejo específico de fluidos para lograr este objetivo es altamente complejo y escapa a los objetivos de esta guía, sin embargo, se hace hincapié en su persecución, para lo cual es esencial la evaluación clínica dirigida, la implementación de medidas de monitorización (sonda Foley, por ejemplo) e inicio inmediato de medidas preventivas o de tratamiento según corresponda. El requerimiento basal habitual para un paciente con HSAa, se estima en aproximadamente $40 \mathrm{ml} / \mathrm{kg}$ de suero fisiológico al $0,9 \%{ }^{29}$.

\subsubsection{Nimodipino}

El nimodipino sigue siendo el único fármaco disponible en nuestro país, que ha demostrado su utilidad en el tratamiento de la HSAa. Inicialmente, dado que su efecto benéfico se atribuyó a una menor incidencia de VE, se le asignó un rol calificado como "preventivo". El mecanismo exacto por el cual disminuye la morbilidad, sin embargo, ha sido posteriormente cuestionado, sugiriéndose muchas propiedades que interrumpirían algunas de las cadenas de sucesos fisiopatológicos que se inician desde la EBI. Desde este punto 


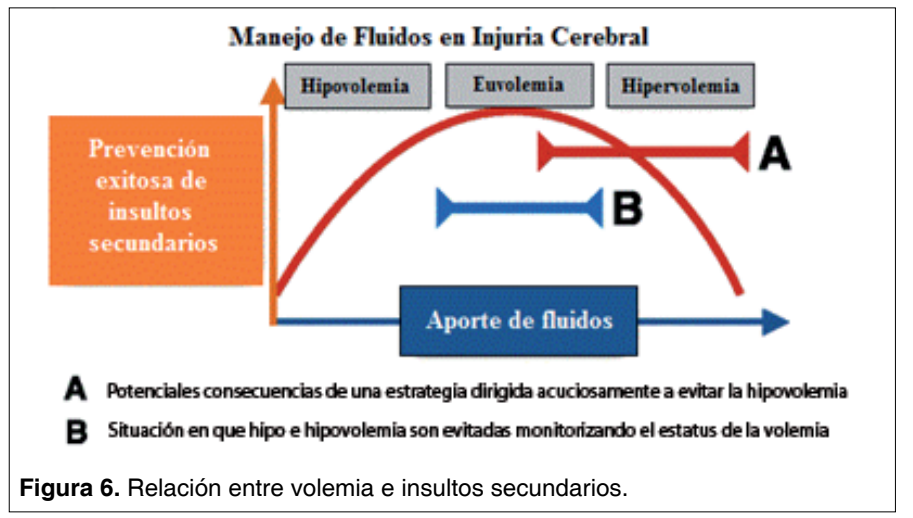

de vista, el nimodipino tendría un rol mucho más "activo" de lo que se creía. Sea como fuere, su efectividad es hoy incuestionable, por lo que debe indicarse a todos los pacientes desde el momento del diagnóstico.

La dosis recomendada es de $60 \mathrm{mg}$ cada 4 h por 21 días, vía oral, excepto en pacientes con inestabilidad hemodinámica, en que su indicación dependerá del análisis de costo/beneficio.

\subsubsection{Tratamiento de la $\mathrm{DCl}$}

\subsubsection{Aumento de la PA (terapia hi-} perdinámica)

Una vez diagnosticada la $\mathrm{DCl}$, la reversión el déficit debe convertirse en un objetivo prioritario. Entre las alternativas terapéuticas, la más accesible en lo inmediato y, por lo tanto, la primera acción a realizar, consiste en intentar aumentar el FSC mediante el aumento de la PA. Esta medida, previamente incluida en la terapia "triple $\mathrm{H}$ ", es el único componente de esa estrategia terapéutica cuya efectividad ha sido respaldada por la evidencia ${ }^{3,4}$. A continuación, se describe un protocolo práctico para el aumento la $\mathrm{PA}^{8,30}$ :

1- Administración de un $15 \mathrm{~mL} / \mathrm{Kg}$ de suero fisiológico $0,9 \%$ en $1 \mathrm{~h}$.

2- Inicio o aumento de infusión continua de noradrenalina, para un aumento escalonado, de a 10 $\mathrm{mmHg}$, en la presión arterial sistólica (PAS), sin sobrepasar los 220 $\mathrm{mmHg}$ de PAS, $140 \mathrm{mmHg}$ de PAM o $120 \mathrm{mmHg}$ de PPC.

3- En caso de revertirse el déficit, mantener el tratamiento por $72 \mathrm{~h}$.

4- En caso de persistir el déficit, evaluar la indicación de angioplastía.

* El avance al siguiente escalón de tratamiento, se produce por la falta de respuesta al tratamiento tras 30 minutos de haberse implementado.

** Durante el tratamiento, se debe monitorizar en forma dirigida la aparición de signos de sobrecarga miocárdica.

*** En base a la literatura más reciente, se considera que la implementación de la terapia hiperdinámica es recomendable incluso en el caso de aneurismas no excluidos ${ }^{31}$.

Además, se sugiere considerar el uso de inótropos (dobutamina o milrinona) en las siguientes situaciones:

- Condición cardiopulmonar contraindica al aumento de PA.

- Angioplastía no disponible.

- Angioplastía no indicada (por ejemplo, DSA sin VE).

\subsubsection{Angioplastía:}

Junto al aumento de la PA, la angioplastía es la única otra medida terapéutica que ha demostrado efectividad en el tratamiento de la DCl. La angioplastía es un tratamiento invasivo y no exento de complicaciones, por lo que no se recomienda su empleo para profilaxis de $\mathrm{VE}^{3,4}$. En cuanto al tipo de angioplastía, ya sea mecánica o farmacológica, y al fármaco a utilizar, no existen diferencias en la literatura, por lo que no es posible hacer una recomendación al respecto.

\section{Recomendaciones para el manejo de las complicaciones sistémicas}

Además de las complicaciones neurológicas ya descritas, el paciente víctima de una HSAa está sujeto a una serie de complicaciones sistémicas asociadas. Si bien algunas de éstas tienen una etiología relacionada con el descalabro neurológico, como la hiponatremia y las complicaciones cardiopulmonares, su manejo está mucho más relacionado con el ámbito de la medicina interna, por lo que son tratadas en este apartado. Por lo mismo, son abordadas sólo de manera superficial, presentando únicamente la recomendación actual de las guías.

6.1. Alteraciones cardiopulmonares: Las comorbilidades cardiovasculares y respiratorias de los pacientes víctimas de una HSAa, dado que comparten muchos factores de riesgo (tabaquismo, hipertensión arterial, etc.), son muy frecuentes. En este sentido, están muchas veces expuestos naturalmente a una mayor incidencia de complicaciones cardiopulmonares durante la hospitalización. Las complicaciones más severas en este ámbito, sin embargo, no serían secundarias a esta predisposición, sino que tendrían una etiopatogenia propia de la $\mathrm{HSAa}$, relacionada con una respuesta autonómica e inflamatoria exagerada.

Se recomienda, por lo tanto, mantener un alto índice de sospecha y monitorizar su aparición. Para esto, se deben realizar electrocardiograma y marcadores de injuria miocárdica en el ingreso ${ }^{4}$. Según la evolución, se debe considerar el seguimiento de los marcadores y el complementar el estudio con un ecocardiograma.

\subsection{Hiponatremia}

La etiología de la hiponatremia en la HSAa puede ser diversa, sin embargo, más frecuentemente sería secundaria a una secreción inapropiada de hormona anti-diurética (SSIADH) y, en segundo lugar, al síndrome perdedor de sal (CSW, por sus siglas en inglés), siendo las demás causas muy infrecuentes. $\mathrm{Si}$ bien existen muchas diferencias clínicas y de laboratorio entre ambas condiciones y, por lo tanto, la aproximación terapéutica es distinta, en la práctica esto no se aplicaría al paciente con HSAa. Las particularidades del paciente cursando con una HSAa son, principalmente, la contraindicación a la restricción de agua libre (mantención de euvolemia) y la evolución generalmente autolimitada de la condición que generó la hiponatremia.

En este contexto, el manejo más apropiado sería la corrección directa de la natremia, independiente de la causa. Basado en estos principios, las recomendaciones actuales en el manejo 
En caso de $\mathrm{Na}<133 \mathrm{mEq} / \mathrm{L}$ o una disminución de $6 \mathrm{mEq} / \mathrm{L}$ en $24-48 \mathrm{~h}$ :

1. $\mathrm{NaCl} 3 \mathrm{~g}$ vía enteral cada $6 \mathrm{~h}$

2. Iniciar infusión de $\mathrm{NaCl} 3 \%$ a $20 \mathrm{~mL} / \mathrm{Hr}$ ev

3. Chequear $\mathrm{Na}$ sérico cada $6 \mathrm{~h}$

a. Si $\mathrm{Na}<130 \mathrm{mEq} / \mathrm{L}$

Aumentar la tasa de infusión en $20 \mathrm{~mL} / \mathrm{Hr}$ (máxima tasa $=80 \mathrm{~mL} / \mathrm{Hr}$ )

Si no estaba recibiendo infusión, iniciar $\mathrm{NaCl} 3 \%$ a $20 \mathrm{~mL} / \mathrm{Hr}$ ev

b. Si Na 130 - $135 \mathrm{mEq} / \mathrm{L}$

Aumentar la tasa de infusión en $10 \mathrm{~mL} / \mathrm{Hr}$ (máxima tasa $=80 \mathrm{~mL} / \mathrm{Hr}$ )

Si no estaba recibiendo infusión, iniciar $\mathrm{NaCl} 3 \%$ a $10 \mathrm{~mL} / \mathrm{Hr}$ ev

c. Si Na 136-140 mEq/L:

No hacer cambios

d. Si $\mathrm{Na}>140 \mathrm{mEq} / \mathrm{L}$

Detener la infusión

Figura 7. Protocolo de manejo de hiponatremia

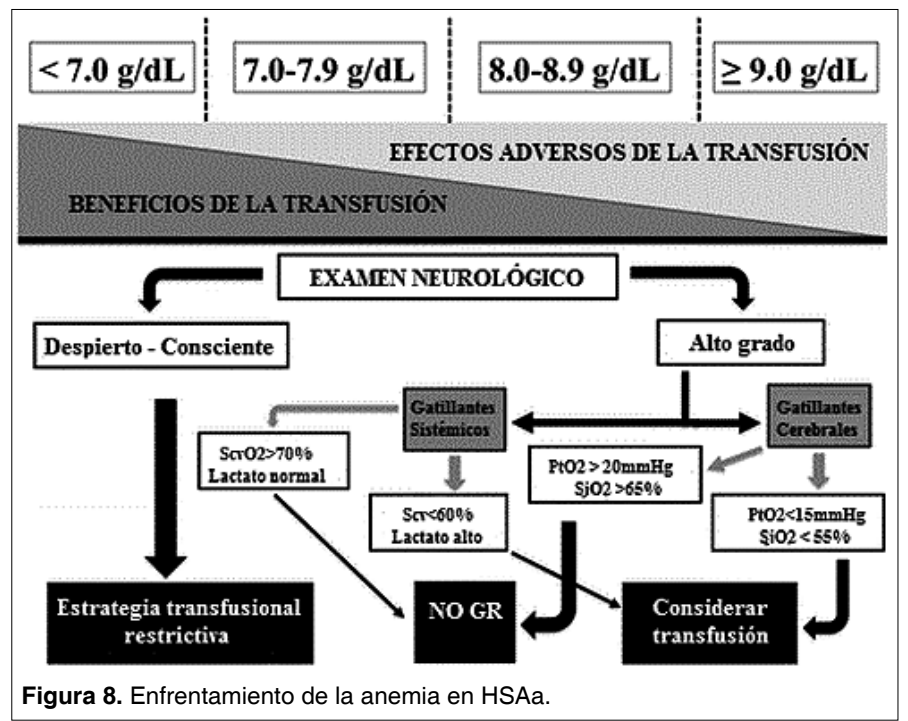

de la hiponatremia son: evitar la restricción de agua, utilizar soluciones hipertónicas y, en caso de persistencia o evidencia de natriuresis aumentada, agregar hidrocortisona o fludrocortisona (por la posibilidad de CSW ${ }^{4}$.

Para evitar el diagnóstico tardío y faci- litar el manejo, se sugiere la implementación del protocolo que se muestra en la Figura $7^{32}$.

\subsection{Anemia}

La mejor conducta ante un descenso en el nivel de hemoglobina $(\mathrm{Hb})$ de un paciente con HSAa es actualmente debatida. Esta controversia nace de una aparente paradoja creada por la evidencia actualmente disponible pues, pese a que existe una fuerte asociación entre anemia y peores outcomes en pacientes con HSAa, éstos también han sido asociados a la transfusión de glóbulos rojos (RBCT, por sus siglas en inglés). Ante esta situación, la recomendación sería no establecer un valor de $\mathrm{Hb}$ predefinido para manejar estos pacientes, sino que indicar la RBCT sólo cuando el paciente lo requiera según parámetros clínicos y de laboratorio. Un esquema que ilustra adecuadamente esta recomendación, se muestra en la Figura $8^{33}$.

\subsection{Enfermedada tromboembólica}

La trombosis venosa es frecuente en pacientes con HSAa, por lo que debe iniciarse inmediatamente la profilaxis mecánica (por ejemplo, compresión neumática intermitente).

Transcurridas $24 \mathrm{~h}$ desde el tratamiento del aneurisma, se debe iniciar la profilaxis farmacológica ${ }^{4}$.

\subsection{Alteraciones de la glicemia}

Tanto la hiperglicemia, como la hipoglicemia, se han asociado a peores outcomes en pacientes neurocríticos. Por este motivo, se debe mantener la glicemia en un rango entre $80-200 \mathrm{mg} /$ $\mathrm{dL}^{4}$.

\subsection{Fiebre}

Se recomienda el tratamiento agresivo de la fiebre, buscando la normotermia, ya sea mediante el uso de fármacos anti-piréticos, o de sistemas de modulación de temperatura ${ }^{3}$.

Recibido: 09 de abril de 2017

Aceptado: 25 de mayo de 2017

\section{Referencias}

1. República de Chile, Ministerio de Salud 2007. Guía Clínica Hemorragia Subaracnoidea Secundaria a Rotura de Aneurismas Cerebrales.

2. Lovelock CE, Rinkel GJ, Rothwell PM. Time trends in outcome of subarachnoid hemorrhage: population-based study and systematic review. Neurology 2010; 74(19): 1494-1501.

3. Connolly ES Jr, Rabinstein AA, Carhuapoma JR, et al Guidelines for the management of aneurysmal subarachnoid hemorrhage: a guideline for healthcare professionals from the American Heart Association/American Stroke Association. Stroke 2012; 43(6): $1711-1737$. 
4. Diringer MN, Bleck TP, Claude Hemphill J 3rd, et al. Critical care management of patients following aneurysmal subarachnoid hemorrhage: recommendations from the Neurocritical Care Society's Multidisciplinary Consensus Conference. Neurocrit Care 2011; 15(2): 211-240.

5. Steiner T, Juvela S, Unterberg A, Jung C, Forsting M, Rinkel G. European Stroke Organization: European Stroke Organization guidelines for the management of intracranial aneurysms and subarachnoid haemorrhage. Cerebrovasc Dis 2013; 35: 93-112.

6. Edlow JA, Caplan LR. Avoiding pitfalls in the diagnosis of subarachnoid hemorrhage. N Engl J Med 2000; 342(1): 29.

7. Suárez JI, Tarr RW, Selman WR. Aneurysmal subarachnoid hemorrhage. N Engl J Med 2006; 354(4): 387-396.

8. Suárez Jl. Diagnosis and Management of Subarachnoid Hemorrhage. Continuum (Minneap Minn). 2015 Oct;21(5 Neurocritical Care): 1263-1287.

9. Siddiq F, Khan A. Perimesencephalic nonaneurysm subarachnoid hemorrhage. In: UpToDate, Post TW (Ed), Up To Date, Waltham, MA. (Accesed on May 15, 2016).

10. Wallace A, Vyhmeister R, Dines $\mathrm{J}$, et al. Evaluation of an anatomic definition of non-aneurysmal perimesencephalic subarachnhoid hemorrhage. J Neurointerv Surg. 2016; 8(4): 378.

11. De Oliveira M, Mansur A, Murphy A, et al. Aneurysmal subarachnoid haemorrhage from a neuroimaging perspective. Critical Care 2014; 18: 557.

12. Edlow J, Fisher J. Diagnosis of subarachnoid hemorrhage. Time to change the guidelines? Stroke. 2012; 43: $2031-2032$.

13. Migdal V, Wu W, Long D, McNaughton C, Ward M, Self W. Risk-benefit analysis of lumbar puncture to evaluate for nontraumatic subarachnoid hemorrhage in adult ED patients. Am J Emerg Med. 2015 Nov; 33(11): 1597-1601.

14. Nagy K, Skagervik I, Tumani H, et al. Cerebrospinal fluid analyses for the diagnosis of subarachnoid haemorrhage and experience from a Swedish study. What method is preferable when diagnosing a subarachnoid haemorrhage? Clin Chem Lab Med 2013, 51: $2073-2086$.

15. Rosen S, Macdonald L. Grading of subarachnoid hemorrhage: modification of the world World Federation of Neurosurgical Societies scale on the basis of data for a large series of patients. Neurosurgery. 2004; 54(3): 566.

16. de Oliveira Manoel AL, Jaja BN, Germans MR, et al. The VASOGRADE: a simple grading scale for prediction of delayed cerebral ischemia after subarachnoid hemorrhage. Stroke. 2015; 46: 1826-1831.

17. Ransom ER, Mocco J, Komotar RJ, et al. External ventricular drainage response in poor grade aneurysmal subarachnoid hemorrhage: effect on preoperative grading and prognosis. Neurocrit Care. 2007; 6: 174-180.

18. Report of World Federation of Neurological Surgeons Committee on a universal subarachnoid hemorrhage scale. J Neurosurg. $1988 \mathrm{Jun}$ 68(6): 985-986.

19. Frontera J, Claassen J, Schmidt JK, et al. Prediction of symptomatic vasospasm after subarachnoid hemorrhage: The modified Fisher scale. Neurosurgery. 2006 Jul; 59(1): 21-7; discussion 21-27.

20. Kapinos G. Redefining secondary injury after subarachnoid hemorrhage in light of multimodal advanced neuroimaging, intracranial and transcranial neuromonitoring: beyond vasospasm. J. Fandino et al. (eds.) Neurovascular events after subarachnoid hemorrhage. Acta Neurochirurgica Supplement. Vol. 120.

21. Sandsmark D, Kumar M, Park S, Levine J. Multimodal monitoring in subarachnoid hemorrhage. Stroke. 2012; 43: 1440-1445.

22. Hillman J, Fridriksson S, Nilsson O, Yu Z, Saveland H, Jakobsson KE. Immediate administration of tranexamic acid and reduced incidence of early rebleeding after aneurysmal subarachnoid hemorrhage: a prospective randomized study. J Neurosurg. 2002; 97(4): 771.

23. Ortega Gutiérrez S, Thomas J, Reccius A, et al. Effectiveness and safety of nicardipine and labetalol infusion for blood pressure management in patients with intracerebral and subarachnoid hemorrhage. Neurocrit care. 2013; 18: 13-19.

24. Glisic $\mathrm{E}$, Gardiner L, Josti L, et al. Inadequacy of headache management after subarachnoid hemorrhage. American Journal of Critical Care. 2016; 25: 136-143.

25 Naidech A, Kreiter K, Janjua N, et al. Phenytoin exposure is associated with Functional and cognitive disability after subarachnoid hemorrhage. Stroke. 2005; 36: 583-587.

26. De Marchis G, Pugin D, Meyers E, et al. Seizure burden in subarachnoid hemorrhage associated with functional and cognitive outcome. Neurology 2016; 86: 1-8.

27. Vergouwen M, Participants in the international multi-disciplinary consensus conference on the critical care management of subarachnoid hemorrhage. Vasospasm versus delayed cerebral ischemia as an outcome event in clinical trials and observational estudios. Neurocrit Care. 2011 Sep; 15(2): 308-311.

28. Wilson CD, Jai J, Shankar S. Diagnosing vasospasm after subarachnoid hemorrhage: CTA and CTP. Can J Neurol Sci. 2014; 41: 314319.

29. Van der Jagt M. Fluid management of the neurological patient: a concise review. Critical Care 2016; $20: 126$.

30. Francoeur C, Mayer S. Management of delayed cerebral ischemia after subarachnoid hemorrhage. Critical Care 2016; $20: 277$.

31. Reynolds M, Buckley R, Indrakanti S, et al. The safety of vasopressor-induced hypertension in subarachnoid hemorrhage patients with coexisting unruptured, unprotected intracranial aneurysms. J Neurosurg. 2015; 123: 862-871.

32. Singer R, Ogilvy C, Rordorf G. Treatment of subarachnoid hemorrhage. In: Up To Date, Post TW (Ed), Up To Date, Waltham, MA. (Accesed on June 30, 2016).

33. Lelubre C, Bouzat P, Crippa I, Taccone F. Anemia management after acute brain injury. Critical Care 2016; 20 : 152.

34. Macdonald RL. Delayed neurological deterioration after subarachnoid haemorrhage. Nat Rev Neurol. 2014 Jan; 10 (1): 44-58.

35. De Oliveira Manoel AL, Goffi A, Marotta TR, Schweizer TA, Abrahamson S, Macdonald RL. The critical care management of poor-grade subarachnoid haemorrhage. Crit Care. 2016 Jan 23; 20-21.

\section{Correspondencia a:}

Dr. Leonidas Quintana Marín

Cátedra de Neurocirugía , Escuela de Medicina

Universidad de Valparaíso, Chile.

leonquin@gmail.com 